\title{
Restoration of solar images by the Steer algorithm ${ }^{\star}$
}

\author{
H. Koshiishi ${ }^{\star \star, \star \star \star}$ \\ National Space Development Agency of Japan, 2-1-1, Sengen, Tsukuba, Ibaraki 305-8505, Japan \\ Received 23 July 2002 / Accepted 5 August 2003

\begin{abstract}
Restoration of diffuse features and computational time have been more serious problems in solar observations than in non-solar observations because of complicated brightness structures in enormous time-series images. The CLEAN algorithm proposed by Steer et al. was applied to the data processing of the Nobeyama Radioheliograph to solve these problems, and restores daily solar images well in an automatic data processing routine. A single set of parameters used in the algorithm can cover a wide range of solar activity. The algorithm also has the advantage of requiring less computational time and is optimized to require one-tenth the time of the classical Högbom algorithm.
\end{abstract}

Key words. instrumentation: interferometers - techniques: image processing - Sun: radio radiation

\section{Introduction}

Since the CLEAN algorithm was first introduced by Högbom (1974) to acquire finer images of radio sources, it has been a powerful method for restoration in aperture-synthesis observations. Limitations of this algorithm have also been discussed many times (e.g. Clark 1980; Cornwell 1983; Cornwell et al. 1999; Schwab 1984; Segalovitz \& Frieden 1978), primarily its failure to restore diffuse features and excessive computational time.

Restoration of diffuse features and computational time have been more serious problems, especially in solar observations. Solar images consist of various components: a solar disk component; some bright compact sources like flares; some faint compact sources like radio emissions associated with $\mathrm{X}$-ray bright points and X-ray jets; and many diffuse features like active regions, prominences, depressions associated with dark filaments, and plateau-shaped enhancements in polar and coronal-hole regions. Complicated structures combining these various components cause restoration failure and increased computational time when using conventional algorithms.

Solar observations also impose another unique condition in data processing, which is an enormous number of time-series images as compared with non-solar observations. For example, 28800 images are acquired during the daily 8-hour operation with $1 \mathrm{~s}$ temporal resolution. Each image can be carefully processed by specified algorithms with manually controlled parameters similarly to what is done in non-solar observations. However, every image must first be processed automatically

\footnotetext{
* Appendix A is only available in electronic form at http://www .edpsciences.org

$\star \star$ e-mail: koshiishi.hideki@nasda.go.jp

$\star \star \star$ Formerly the University of Tokyo.
}

without any parameter adjustment in order to determine when and where remarkable phenomena occur. Initial data processing in solar observations, therefore, requires an ability to process data with a single set of parameters that can cover a wide range of solar activity.

To solve these problems, the CLEAN algorithm proposed by Steer et al. (1984) (the Steer algorithm hereafter) was applied to the data processing of the Nobeyama Radioheliograph (the NoRH hereafter; a radio interferometer at $17 \mathrm{GHz}$ and $34 \mathrm{GHz}$ dedicated to solar observations) of the Nobeyama Solar Radio Observatory, the National Astronomical Observatory of Japan (Enome 1996; Koshiishi 1996; Nakajima et al. 1994; Nishio et al. 1994; Takano et al. 1994). The capability of the Steer algorithm is, however, not yet fully understood (e.g. Cornwell et al. 1999). This paper discusses the performance of the Steer algorithm in restoring solar images acquired by the NoRH.

\section{Restoration of solar images by the Steer algorithm}

The Steer algorithm deals with radio sources in a group rather than individually. Components brighter than a certain fraction of the peak brightness in the dirty image are regarded as a group, called a contour-trim. The fraction of the contour-trim must exceed the normalized level of the largest sidelobe. The contour-trim is convolved with the dirty beam to create a dirty contour-trim, which is extracted from the dirty image with a CLEAN loop gain. The NoRH restoration procedure uses 0.8 for the fraction of the contour-trim, 0.2 for a CLEAN loop gain, and $3 \sigma$ for a CLEAN criterion where $\sigma$ is the rms noise level in images (see Appendix). 

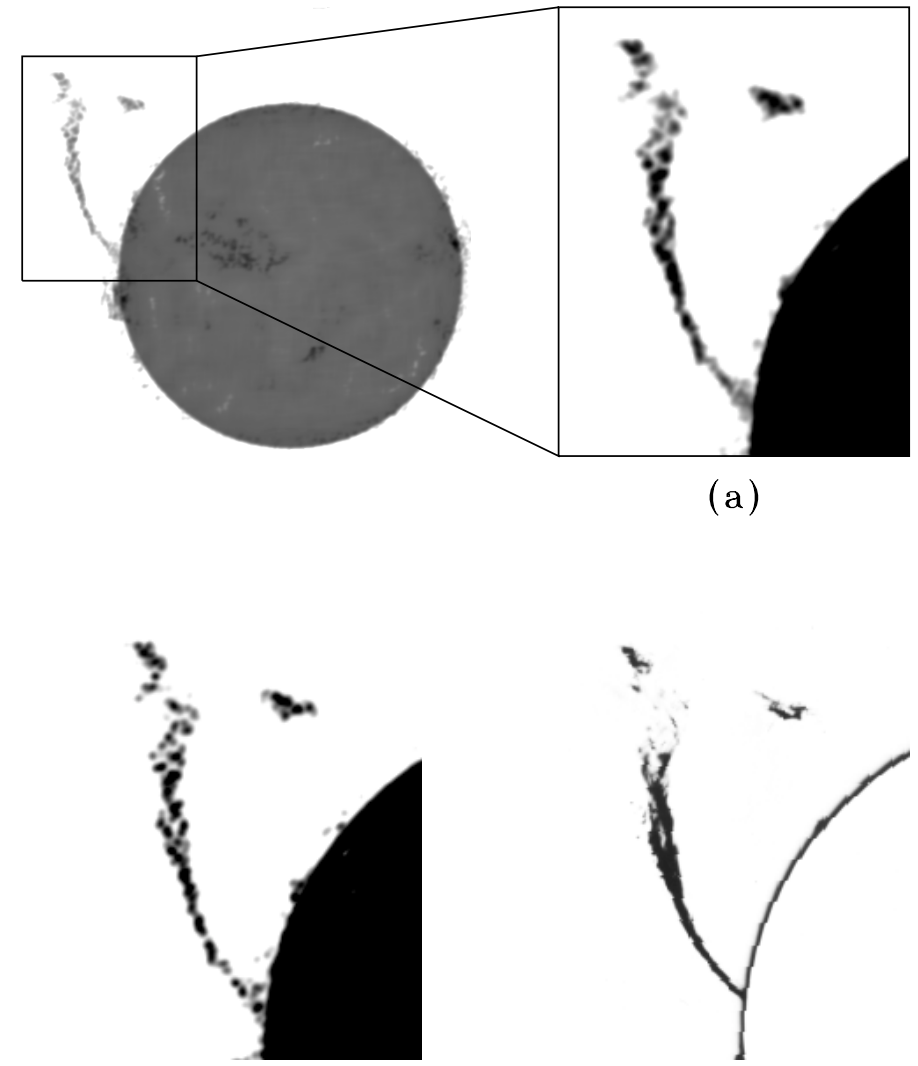

(b) (a)

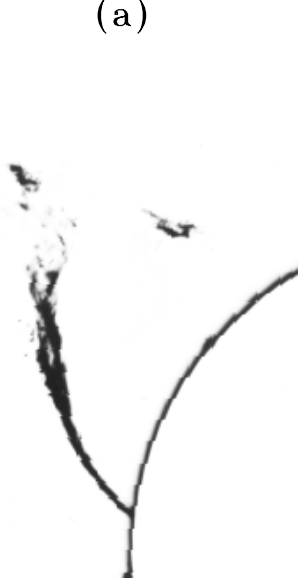

(c)

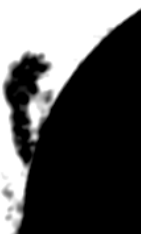

$(\mathrm{d}-1)$

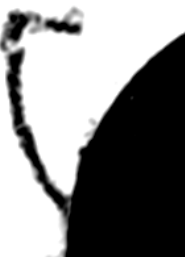

$(d-4)$

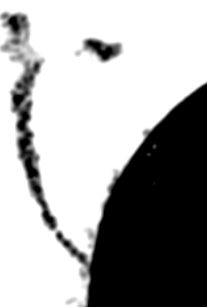

$(d-7)$

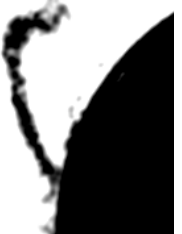

$(d-2)$

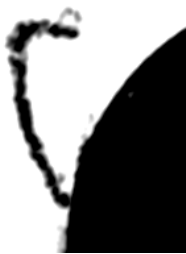

$(d-3)$
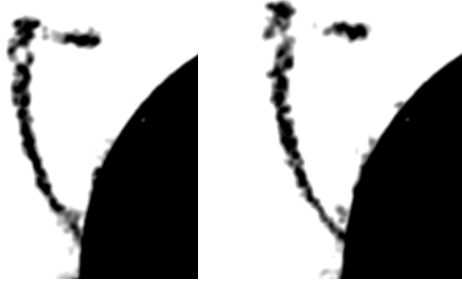

$(d-5)$

$(d-6)$

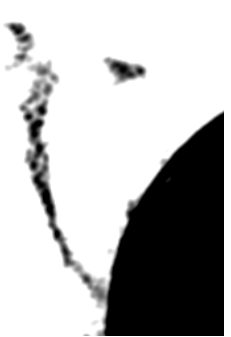

$(d-8)$

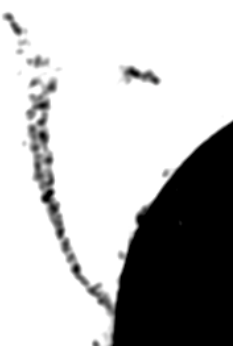

$(d-9)$

Fig. 1. Successive observations by the Nobeyama Radioheliograph of an eruptive prominence that occurred on 31 July 1992 above the east solar limb. a) is a partial image of the whole Sun at 01:15:41 (UT) processed by the Steer algorithm. The FOV is $16 \times 21$ arcmin. b) is an image obtained from the same data as a), however is processed by the classical Högbom algorithm. c) is the same prominence as a) in $\mathrm{H} \alpha$ at the same time with almost the same FOV observed by the Norikura $10 \mathrm{~cm}$-diameter Coronagraph. d-1)-d-9) are successive images processed by the Steer algorithm. The FOV is the same as a). d-1) is 00:15 (UT). d-2) is 00:45 (UT). d-3) is 00:50 (UT). d-4) is 00:55 (UT). d-5) is 01:00 (UT). d-6) is 01:05 (UT). d-7) is 01:10 (UT). d-8) is 01:15 (UT). d-9) is 01:20 (UT).

\subsection{Performance of the Steer algorithm}

The rms differential level is often used in simulations to quantitatively discuss fidelity of restoration. An aspect of restoration of weak diffuse features is, however, buried in the rms quantity taken for a large number of pixels. Furthermore, results of restoration strongly depend on models used in simulations. Solar observations, fortunately, make it possible to take a movie of features in motion. To discuss the fidelity of the Steer algorithm, time-series images are compared with each other.

Among the variety of phenomena on the Sun, eruptive activity is one of the most dynamic events. Figure 1 depicts successive observations by the NoRH of an eruptive prominence that occurred on 31 July 1992 above the east solar limb. Untwisting motion of two major threads, which cannot be separately distinguished at the beginning of eruption, are seen clearly. Comparison with an $\mathrm{H} \alpha$ image, taken by the Norikura $10 \mathrm{~cm}$-diameter Coronagraph of the Norikura Solar Observatory, shows a complementary view. In contrast, the two threads are hardly distinguishable in the image processed by the classical Högbom algorithm.

In the early phase of eruption, the two threads are tightly twisted and retain high brightness. As the untwisting motion proceeds, the brightness of the two threads decreases. Stripes caused by phase errors in the calibrated visibilities (see Appendix) enhance or reduce the brightness of intersecting points between stripes and the two threads in the dirty image. The restoration procedure then produces graininess seen in the last phase of eruption, which is not due to the performance of the restoration procedure itself.

Studies of eruptive events using the NoRH data processed by the Steer algorithm have also been conducted by, for example, Gopalswamy et al. (1997), Gopalswamy \& Hanaoka (1998), Hanaoka \& Shinkawa (1999), Hori et al. (2000), and Hori (2000). Figure 1 and the observational results in these studies demonstrate that the Steer algorithm can restore complicated structures well with the single set of parameters, at least as first-step images.

Additionally, the Steer algorithm used 22 iterations to process the image in Fig. 1a while 1083 iterations were required by the classical Högbom algorithm with the same CLEAN loop gain and the same CLEAN criterion. The Steer algorithm includes FFTs in each iteration so that computational time of each iteration exceeds that of the classical Högbom algorithm. In spite of this, the Steer algorithm still has the advantage of 


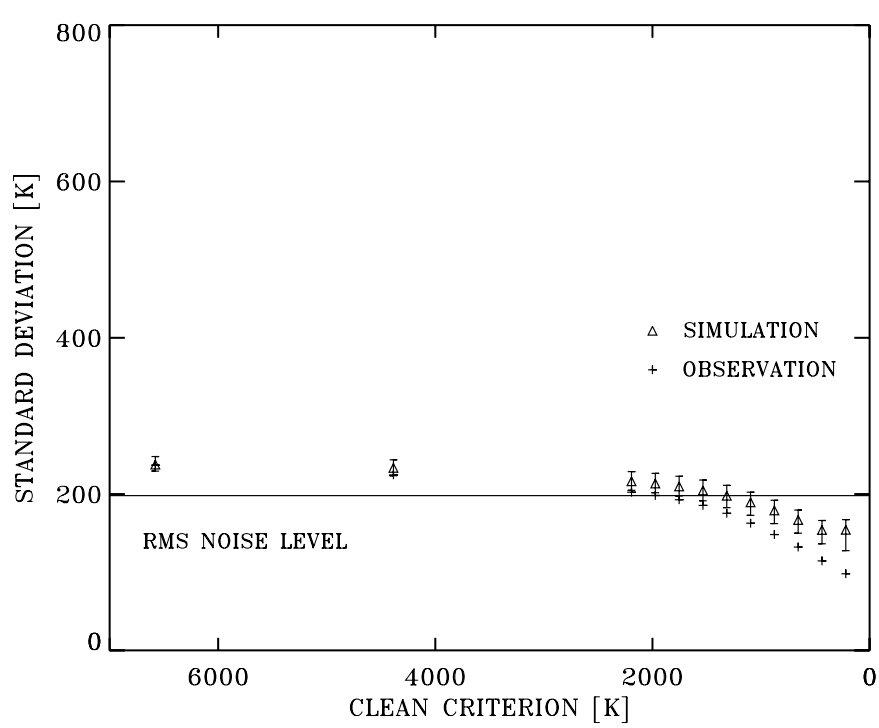

Fig. 2. The rms noise level, and the rms level in the sky region excluding CLEAN points in the simulated image and in the observed image of the Nobeyama Radioheliograph at each CLEAN criterion.

requiring less computational time. The actual total time for restoration is one-tenth the time of the classical Högbom algorithm under the same calculation conditions.

\subsection{Very elongated features below the CLEAN criterion}

Below the CLEAN criterion, radio sources are contaminated by sidelobe responses of other non-restored components. If radio sources above the CLEAN criterion are restored well, specific radio sources like very elongated features, which are below the CLEAN criterion, are also available for study. The average brightness of available features must exceed the largest sidelobe response of non-restored components, $1.5 \sigma$ in the NoRH case. This is due to the following reasons.

Noise is present over the whole image. The CLEAN algorithm intrinsically concentrates noise from the whole image into peak-brightness points of several CLEAN components (CLEAN points hereafter). The practical noise level is thus lower than the rms noise level except for several CLEAN points. Figure 2 presents the rms level in the sky region excluding CLEAN points in the simulated image (see Appendix) and in the observed image of the NoRH at each CLEAN criterion. Both rms levels decrease from the rms noise level by 20 percent at a CLEAN criterion of $3 \sigma$. Figure 3 illustrates the noise concentration and the image smoothing in the observed image of the NoRH with two CLEAN criteria.

In addition, broad aspects of very elongated features are hardly contaminated by the noise concentration and the sidelobe responses of other non-restored components because most of the shapes of these features are much larger, although fine structures are severely disturbed. Very elongated features are also little affected by the dilution effect since these features are sufficiently larger than the mainlobe of the dirty beam.

Figure 4 depicts an example of very elongated features that are below the CLEAN criterion and brighter than the largest

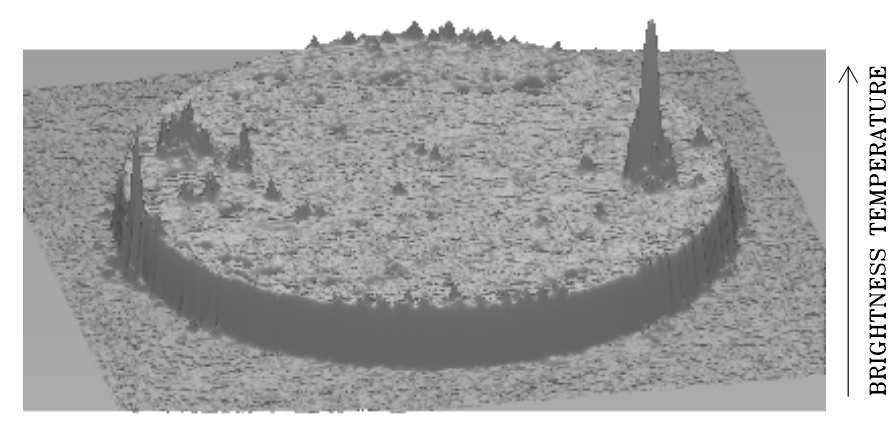

(a)

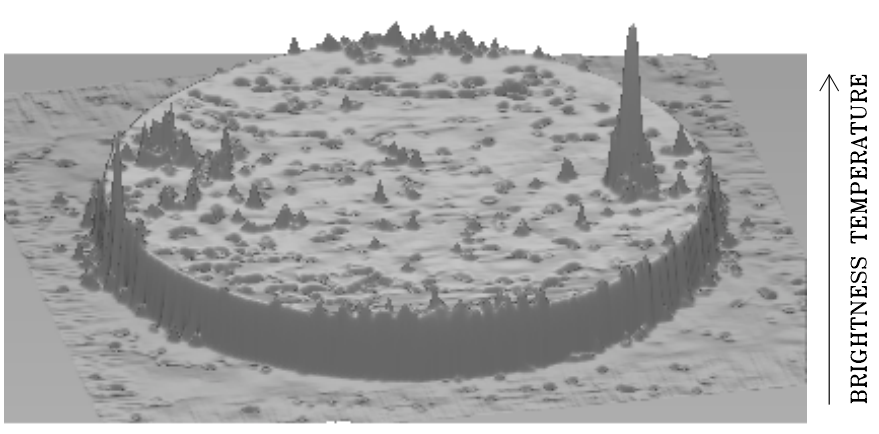

(b)

Fig. 3. Noise concentration and image smoothing in the observed image of the Nobeyama Radioheliograph with two CLEAN criteria. CLEAN criterion of a) is $3 \times \mathrm{rms}$ noise level. CLEAN criterion of $\mathbf{b}$ ) is $0.5 \times$ rms noise level.

sidelobe response of non-restored components. $17-\mathrm{GHz}$ enhanced emissions of $500 \mathrm{~K}$ or more from the quiet disk brightness of $10000 \mathrm{~K}$ observed by the NoRH are overlaid with soft X-ray images acquired by the Soft X-ray Telescope (SXT) on board the YOHKOH satellite (Tsuneta et al. 1991). Corotations of the $17-\mathrm{GHz}$ enhanced emissions with southernpolar and middle-latitude elongated coronal holes in the soft $\mathrm{X}$-ray are easily seen in 4-day consecutive images. In contrast, it is difficult to distinguish these association in the image processed by the classical Högbom algorithm.

Very elongated features observed by the NoRH and processed by the Steer algorithm have also been studied by, for example, Shibasaki (1998) and Gopalswamy et al. (1999). Figure 4 and the observational results in these studies demonstrate that, below the CLEAN criterion, specific radio sources like very elongated features brighter than the largest sidelobe response of non-restored components become available for study by the Steer algorithm.

\section{Conclusions}

The Steer algorithm applied to the NoRH data processing restores complicated brightness structures well in daily solar images in the automatic data processing routine. The single set of parameters used in the Steer algorithm can cover a wide range of solar activity. The Steer algorithm also has the advantage of requiring less computational time and is optimized to require one-tenth the time of the classical Högbom algorithm. 


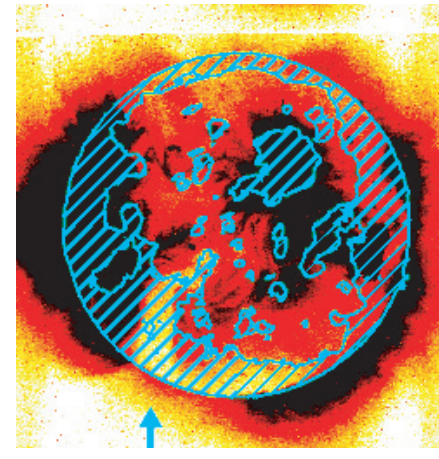

(a)

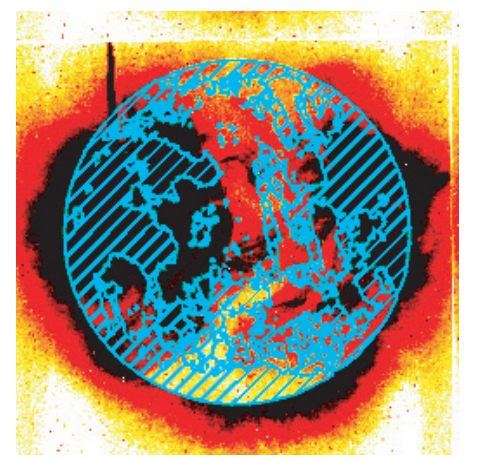

(e)

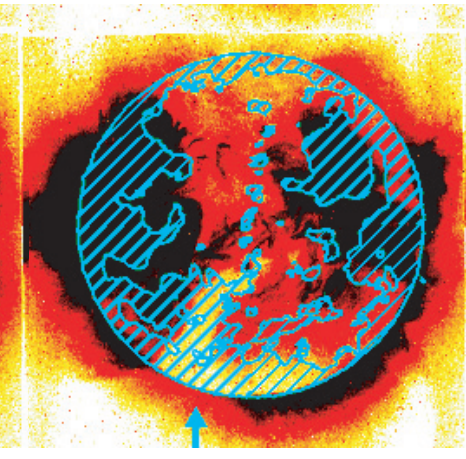

(b)

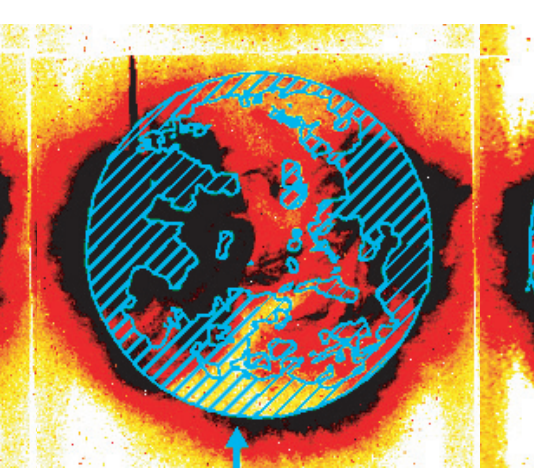

(c)

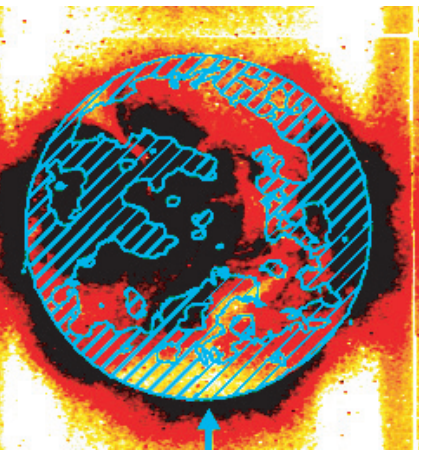

(d)

Fig. 4. Consecutive observations of $17-\mathrm{GHz}$ enhanced emissions associated with soft X-ray coronal holes, which are indicated by blue arrows. Color-scaled images are the soft X-ray emission observed by the Soft X-ray Telescope. Blue contours with stripes are the 17-GHz enhanced emissions of $500 \mathrm{~K}$ or more from the quiet disk brightness of $10000 \mathrm{~K}$ observed by the Nobeyama Radioheliograph. a)-d) are 4-day consecutive images around noon from 4 March 1993 through 7 March 1993, and are processed by the Steer algorithm. e) is obtained from the same data as $\mathbf{c}$ ), however is processed by the classical Högbom algorithm.

Acknowledgements. The author would like to express his gratitude to S. Enome, K. Shibasaki, and H. Nakajima for fruitful discussions. The author is also grateful to T. Bastian for useful comments. Special thanks are due to T. Sakurai, H. Miyazaki, and M. Irie for providing $\mathrm{H} \alpha$ images of the eruptive prominence taken by the Norikura $10 \mathrm{~cm}$-diameter Coronagraph of the Norikura Solar Observatory, the National Astronomical Observatory of Japan. All YOHKOH satellite members of the Institute of Space and Astronautical Science are also acknowledged for soft X-ray images of the whole Sun acquired by the Soft X-ray Telescope.

\section{References}

AIPS Cook Book

Clark, B. G. 1980, A\&A, 89, 377

Cornwell, T. J. 1983, A\&A, 121, 281

Cornwell, T., Braun, R., \& Briggs, D. S. 1999, in Synthesis Imaging in Radio Astronomy II, ed. G. B. Taylor, C. L. Carilli, \& R. A. Perley, ASP Conf. Ser., 180, 151

Enome, S. 1996, in Magnetodynamic Phenomena in the Solar Atmosphere, ed. Y. Uchida, T. Kosugi, \& H. S. Hudson, Proc. IAU Colloq., 153, 195

Fujiki, K. 1998, Ph.D. Dissertation, the Graduate University for Advanced Studies

Gopalswamy, N., Hanaoka, Y., Kundu, M. R., et al. 1997, ApJ, 475, 348

Gopalswamy, N., \& Hanaoka, Y. 1998, ApJ, 498, L179

Gopalswamy, N., Shibasaki, K., Thompson, B. J., et al. 1999, JGR, 104,9767
Hanaoka, Y., Shibasaki, K., Nishio, M., et al. 1994, in New Look at the Sun with Emphasis on Advanced Observations of Coronal Dynamics and Flares, ed. S. Enome, \& T. Hirayama, NRO Report, 360,35

Hanaoka, Y., \& Shinkawa, T. 1999, ApJ, 510, 466

Högbom, J. A. 1974, A\&AS, 15, 417

Hori, K., Kosugi, T., Fujiki, K., et al. 2000, ApJ, 533, 557

Hori, K. 2000, ApJ, 543, 1011

Jennison, R. C. 1958, MNRAS, 118, 276

Koshiishi, H., Enome, S., Nakajima, H., et al. 1994, PASJ, 46, L33

Koshiishi, H. 1996, Ph.D. Dissertation, the University of Tokyo

Nakajima, H., Sekiguchi, H., Aiba, S., et al. 1980, PASJ, 32, 639

Nakajima, H., Nishio, M., Enome, S., et al. 1994, Proc. IEEE, 82, 705

Nishio, M., Nakajima, H., Enome, S., et al. 1994, in New Look at the Sun with Emphasis on Advanced Observations of Coronal Dynamics and Flares, ed. S. Enome, \& T. Hirayama, NRO Report, 360, 19

Noordam, J. E., \& de Bruyn, A. G. 1982, Nature, 299, 597

Perley, R. A. 1999, in Synthesis Imaging in Radio Astronomy II, ed. G. B. Taylor, C. L. Carilli, \& R. A. Perley, ASP Conf. Ser., 180, 275

Schwab, F. R. 1984, AJ, 89, 1076

Segalovitz, A., \& Frieden, B. R. 1978, A\&A, 70, 335

Shibasaki, K. 1998, in Synoptic Solar Physics, ed. K. S. Balasubramaniam, J. W. Harvey, \& D. M. Rabin, ASP Conf. Ser., 140,373

Steer, D. G., Dewdney, P. E., \& Ito, M. R. 1984, A\&A, 137, 159

Takano, T., Enome, S., Nakajima, H., et al. 1994, in Astronomy with Millimeter and Submillimeter Wave Interferometry, ed. M. Ishiguro, \& W. J. Welch, ASP Conf. Ser., 59, 430

Tsuneta, S., Acton, L., Bruner, M., et al. 1991, Sol. Phys., 136, 37 


\section{Online Material}


H. Koshiishi: Restoration of solar images by the Steer algorithm, Online Material p 2

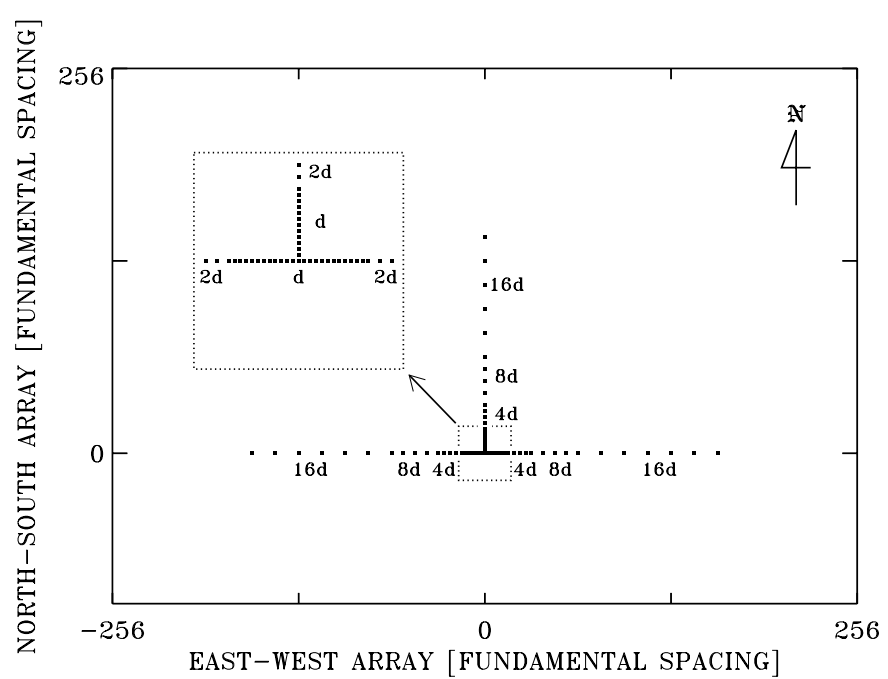

Fig. A.1. The array configuration of the Nobeyama Radioheliograph.

\section{Appendix A: Imaging performance of the Nobeyama radioheliograph}

\section{A.1. Instruments and data processing}

The NoRH consists of $8480 \mathrm{~cm}$-diameter antenna elements. The array configuration is a two-dimensional, multiple equallyspaced, T-shaped array, and has high redundancy. The antenna elements are placed with increasing antenna spacing of $\mathrm{d}$ (fundamental spacing), $2 \mathrm{~d}, 4 \mathrm{~d}, 8 \mathrm{~d}$, and $16 \mathrm{~d}$ from the phase center. Figures A.1 and A.2 illustrate the array configuration and the observing points (UV pattern) in the Fourier domain (UV plane), respectively. The fundamental spacing $\mathrm{d}$ is $86.6459 \lambda$ at $17 \mathrm{GHz}$, corresponding to an interferometer's field of view of about 40 arcmin, which is wide enough to cover the whole Sun. Expected spatial resolution based on the longest antenna spacing is about 10 arcsec. Temporal resolution is $1 \mathrm{~s}$ for snap-shot images and 100 millisec for selected events. Complex visibilities of images of the whole Sun at $17 \mathrm{GHz}$ and of partial images including flaring regions at $34 \mathrm{GHz}$ are obtained for 8 hours a day.

The obtained visibilities are first calibrated by an unique real-time self-calibration method in order to prevent interruption of solar observations. The Sun itself is regarded as a calibrator by considering the redundancy of the array configuration (Nakajima et al. 1980; Noordam \& de Bruyn 1982). Images are then synthesized from the calibrated visibilities on the array plane by a FFT procedure. The dirty beam has many sidelobes due to the array configuration. The normalized level of the largest sidelobe exceeds 0.5 . The dirty image is thus strongly corrupted by its own aliasing.

The restoration procedures are described in the next subsection in detail. After restoration, the restored image is projected on the sky plane, where solar north is to the top and pixel size is 4.91 arcsec square. Finally, gain degradation due to the beam of the antenna elements is corrected. The brightness in images is also scaled by assuming the quiet disk brightness to be $10000 \mathrm{~K}$.

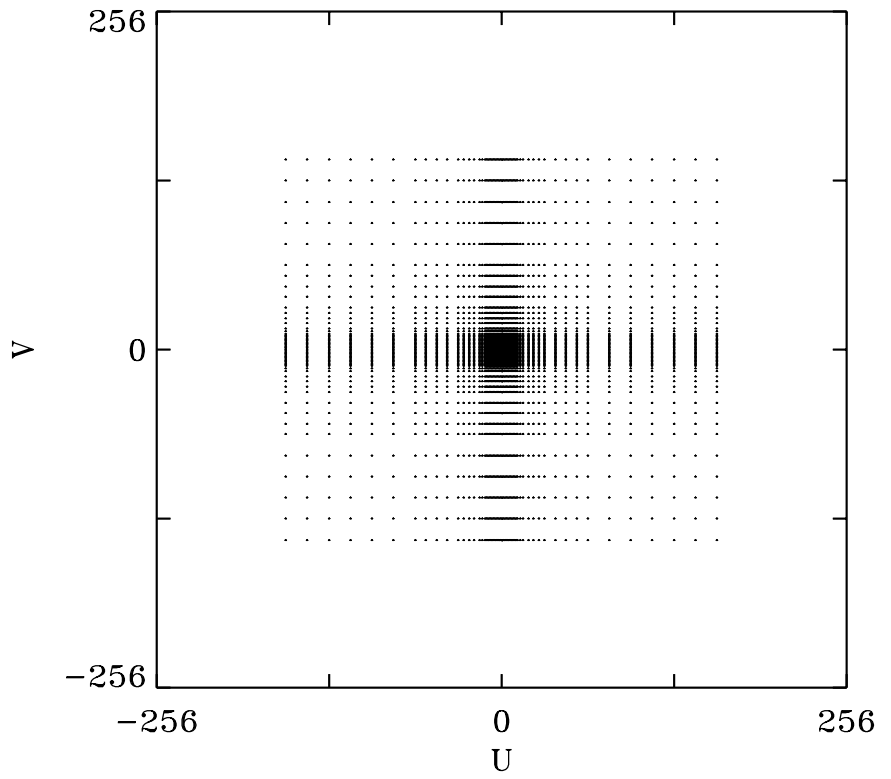

Fig. A.2. The observing points (UV pattern) in the Fourier domain (UV plane) of the Nobeyama Radioheliograph.

\section{A.2. Restoration}

The CLEAN algorithm was applied to the NoRH data processing, and modified to achieve suitable accuracy and efficiency. In the $17-\mathrm{GHz}$ restoration procedure, the disk component and the other sources are extracted separately. A dirty disk is created by convolution of a model disk and the dirty beam, and is extracted from the dirty image at one time. Three restoration procedures are provided for each purpose of data processing to extract some bright and faint compact sources and many diffuse features. For diffuse features, the Steer algorithm described in Sect. 2 is applied.

The procedure optimized to flares (Hanaoka et al. 1994) uses dirty models, which mean the dirty images of model Gaussian sources with various sizes. The brightness distribution near the peak-brightness point in the dirty image is compared to those of the dirty models, and the most appropriate dirty model is extracted with a CLEAN loop gain of 0.02 .

The practical spatial resolution is worse than the expected resolution based on the longest antenna spacing due to the array configuration. To attain the expected resolution in partial images, Fujiki (1998) introduces two-step CLEANs, the first of which processes images of the whole Sun by the Steer algorithm without restoring the disk component. The second one then re-samples only the visibilities of the analysis target in the Fourier domain with a weighting function so as to make the density of the observing points uniform, and processes the analysis target.

After extraction, the restoration procedure convolves the processed components with a CLEAN beam and restores them in the residual image. The CLEAN beam is a Gaussian beam fitted to the mainlobe of the dirty beam. 
H. Koshiishi: Restoration of solar images by the Steer algorithm, Online Material p 3

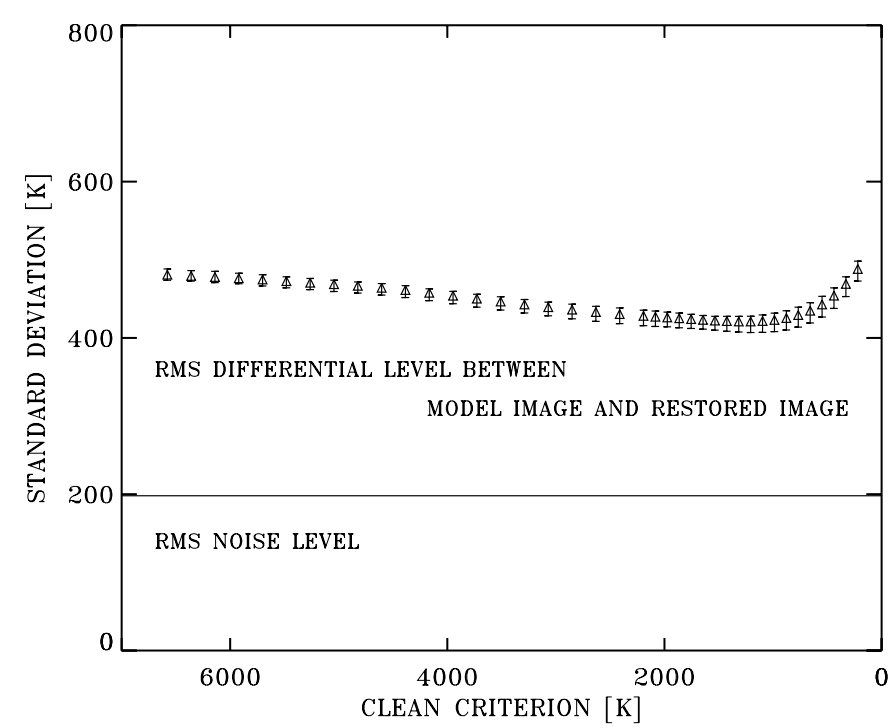

Fig. A.3. The rms noise level in images due to major hardware error sources, and the rms differential level between the model image and the restored image at each CLEAN criterion in the simulation. The error sources are unequal bandpass characteristics, antenna-pointing errors, delay errors, and noise at correlator outputs.

\section{A.3. Imaging Performance}

This section describes the available CLEAN criterion and the rms noise (error) level, which dominate the imaging performance, in snap-shot images at $17 \mathrm{GHz}$ of the NoRH.

\section{A.3.1. Available CLEAN criterion}

The rms noise level in images is the lower limit for detecting true features, and is usually an appropriate CLEAN criterion. Further consideration about the CLEAN criterion is necessary for solar images that are more complex combinations of various-size components than non-solar images.

To evaluate the rms noise level, a whole solar image processed by AIPS (see the AIPS Cook Book) is adopted as a model image in simulations. The visibilities of the model image are calculated at the observing points in the Fourier domain. Influences of major hardware error sources are incorporated into the visibilities. The error sources are unequal bandpass characteristics, antenna-pointing errors, delay errors, and noise at correlator outputs (Koshiishi et al. 1994). Two dirty images are then re-synthesized from the original visibilities and from the visibilities including the influences of the error sources. The rms noise level is calculated by taking the difference of the two dirty images.

Other rms quantities are also calculated to evaluate the available CLEAN criterion. Figure A.3 presents the rms noise level in images and the rms differential level between the model image and the restored image at each CLEAN criterion in the simulation. As restoration proceeds, the rms differential level decreases gradually until the peak brightness in the residual image is comparable to $3 \sigma$. Below this, restoration is affected by noise and the rms differential level rapidly increases. The available CLEAN criterion is, therefore, $3 \sigma$.

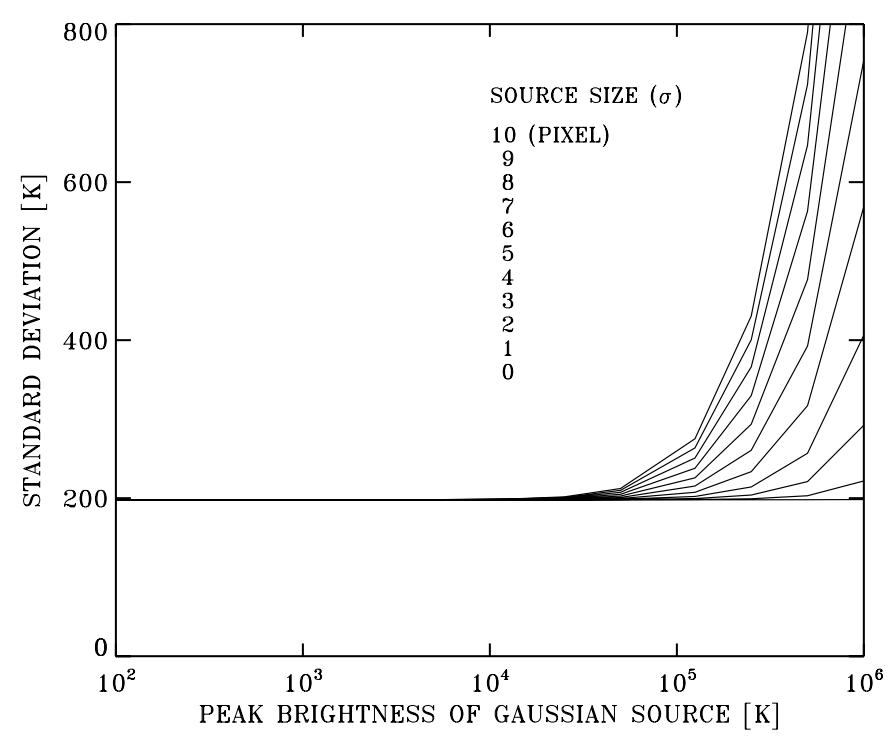

Fig. A.4. Increase of the rms noise (error) level assuming the disk component and a single Gaussian source with various sizes in the simulation. One pixel corresponds to 4.91 arcsec in images.

\section{A.3.2. Noise (Error) level and solar activity}

The error sources can be divided into two categories. Noise is due to finite bandwidth and finite integration time, and added to the obtained visibilities at correlator outputs. The quantity of noise produced in images is stable and independent of solar activity. Influences of unequal bandpass characteristics, antennapointing errors, and delay errors, namely correlator-based errors, appear as a certain fraction in the obtained visibilities, so that the quantity of errors produced in images strongly depends on solar activity.

High Solar Activity Case: When solar activity is high, bright compact sources have large amplitudes of visibilities both in the inner portion of the Fourier domain (corresponding to lower spatial frequencies), and in the outer portion (corresponding to higher spatial frequencies). The high signal-to-noise ratio of the obtained visibilities leads to good self-calibration. For example, closure relations (Jennison 1958) obtained for the redundant longest antenna spacing at the same time show phase fluctuations of less than 1 degree rms in the case of GOES M-class or larger flare occurrence. Phase and amplitude fluctuations in closure relations, however, still cause phase and amplitude errors in the calibrated visibilities, most of which are due to the correlator-based errors.

Since the influences of the correlator-based errors appear as a certain fraction in the obtained visibilities, large amplitudes of visibilities induce numerous amplitude errors. Large flares or many active regions thus increase the quantity of errors produced in images and make it harder to analyze faint sources. Figure A. 4 presents the increase of the rms noise (error) level in the simulation assuming the disk component and a single Gaussian source with various sizes, and considering the influences of the error sources. The rms noise (error) level is stable up to the peak brightness of a single Gaussian source of $10^{5} \mathrm{~K}$, where noise at correlator outputs is dominant, then rises due 


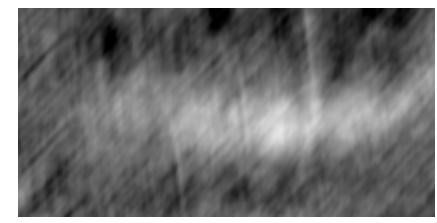

$(\mathrm{a}-1)$

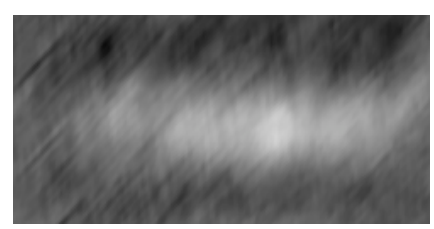

$(b-1)$

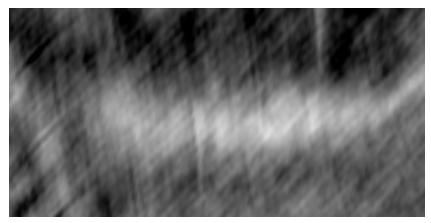

$(a-2)$

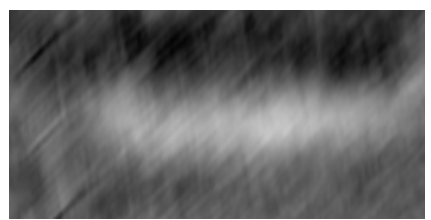

$(b-2)$

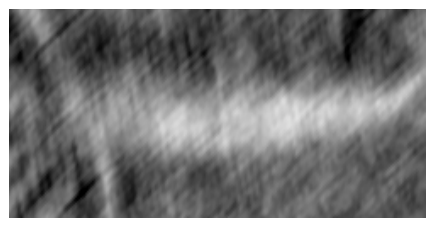

$(a-3)$

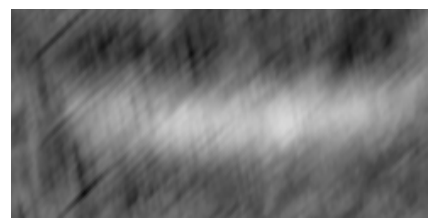

$(b-3)$

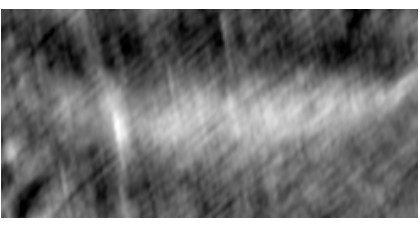

$(\mathrm{a}-4)$

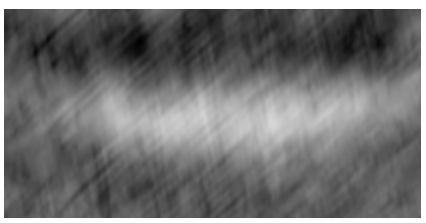

$(b-4)$

Fig. A.5. a-1)-a-4) are successive images of the Nobeyama Radioheliograph at 90-min intervals without the disk component, i.e. 17-GHz enhanced emissions in the south polar region. The FOV is $10 \times 20$ arcmin. Stripes flow from left to right through the FOV with time. b-1)-b-4) are successive images obtained from the same data as $\mathbf{a - 1}$ )-a-4). Components outside the FOV are, however, restored beforehand using the NON-CLEAN BOX.

to the correlator-based errors as the peak brightness increases. Only one compact source is assumed in the simulation so that the actual case is often worse.

The ratio of the rms noise (error) level to the peak brightness in images is defined as the dynamic range of an image, and is better than 1:300 (Koshiishi et al. 1994).

Low Solar Activity Case: When solar activity is low, the major contribution to the visibilities originates only from the disk component. The visibilities have large amplitudes in the inner portion of the Fourier domain while the noise level is comparable to the amplitudes of visibilities in the outer portion. The disk component and diffuse features are well self-calibrated, however faint compact sources are not. For example, closure relations obtained for the redundant longest antenna spacing at the same time show phase fluctuations of several tens of degrees if there are no flares. Large phase errors are then contained in the calibrated visibilities in the outer portion, most of which are induced by noise at correlator outputs. The ratio of the rms noise level to the quiet disk brightness in images is evaluated to be 1:40.

Phase errors in the calibrated visibilities are serious problems in interferometric observations (e.g. Perley 1999). Phase errors cause positional inaccuracies of radio sources, and also produce stripes in images. Stripes enhance or reduce the brightness of intersecting points between stripes and real structures in the dirty image. The restoration procedure often emphasizes this, and leads to misunderstanding of the structures. Furthermore, stripes have directionality determined by the observing points in the Fourier domain, and rotate with the Sun's diurnal motion, although the self-calibration is performed in each snap-shot image. Figure A.5a depicts an example of stripe-flow over faint compact sources with time. The flow of stripes causes fluctuations in $17-\mathrm{GHz}$ enhanced emissions in the south polar region known as polar-cap brightenings, which leads to misunderstanding of the temporal variations.
The visibilities can be integrated to suppress phase errors. The integration does not mean super-synthesis but an integration at the same observing point in the Fourier domain because phase errors arise at each observing point in the case of both snap-shot and super-synthesis imaging. The integration time must thus be shorter than the period through which the observing points rotate to the next grid points.

\section{A.3.3. NON-CLEAN BOX}

The CLEAN BOX, where only components within a specific area are restored, is a reasonable method to avoid producing false features. Solar images, however, consist of many components that need a lot of CLEAN BOXes, so that it is not used in the NoRH data processing.

The effects of sidelobe responses of other sources on bright compact sources rarely restore false features. On diffuse features, even if faint, the effects are less than those on compact sources. The restoration procedure performs well for both components without the CLEAN BOX. In contrast, faint compact sources are easily contaminated by the effects during restoration.

A different method is thus required, the NON-CLEAN BOX, where components outside a specific area are restored beforehand. Figure A.5b depicts the effectiveness of the NON-CLEAN BOX at suppressing the effects of sidelobe responses of outside sources. Faint compact sources inside the NON-CLEAN BOX can, therefore, be properly restored.

\section{A.4. Summary}

In NoRH snap-shot images at $17 \mathrm{GHz}$ when solar activity is high, the dynamic range of an image is better than 1:300. When solar activity is low, the ratio of the rms noise level to the quiet disk brightness is 1:40. The available CLEAN criterion is $3 \sigma$. 